\title{
Superconducting nanowires as quantum phase-slip junctions
}

\author{
J. E. MOOIJ* AND YU. V. NAZAROV \\ Kavli Institute of Nanoscience, Delft University of Technology, 2628 CJ Delft, The Netherlands \\ *e-mail: j.e.mooij@tudelft.nl
}

F or a superconductor, charge and phase are dual quantum variables. A phase-slip event in a superconducting nanowire changes the phase difference over the wire by $2 \pi$; it is the dual process to Cooper-pair tunnelling in a Josephson junction. Phase slip by thermal activation at high temperatures is well understood ${ }^{1}$. Phase slip by quantum tunnelling at low temperatures is considered plausible ${ }^{2,3}$, but experiments on the resistance of nanowires ${ }^{4,5}$ are inconclusive on this point. Büchler et al. ${ }^{6}$ conclude that successive quantum phase slip (QPS) events can be coherent. Here, we demonstrate that, if it exists, coherent QPS is the exact dual to Josephson tunnelling. A narrow nanowire should act as a QPS junction that shows kinetic capacitance, a plasma resonance and current plateaus of interest for nanoelectronic applications. We suggest feasible experiments to unequivocally confirm the existence for coherent QPS.

Phase slip in a thin superconducting wire occurs on the scale of the superconducting coherence length. Phase slip by thermal activation ${ }^{1}$ is observed as a resistive tail below the critical temperature. In wires with diameter below $10 \mathrm{~nm}$ and very high resistance, the energy barrier is small enough that phase slip by quantum tunnelling can be expected ${ }^{2,3}$. Wires of Mo-Ge deposited on suspended carbon nanotubes have been studied ${ }^{3-5}$, and the results are in reasonable agreement with microscopic calculations of phase-slip rates ${ }^{7,8}$. All experiments consisted of passing a small d.c. current through the sample and measuring the voltage. As each phase-slip event in the presence of a current $I$ releases an energy $I \Phi_{0}$, where $\Phi_{0}=h / 2 e$ is the flux quantum, with $h$ the Planck constant and $e$ the electron charge, such measurements are dissipative. Unambiguous experimental evidence of QPS is still absent. It has been concluded ${ }^{5}$ that results can be described by thermally activated phase slip for wires with larger cross-section, and as mesoscopic diffusive normal-metal conductance for the weaker wires. The theoretical analysis is complicated by the fact that the behaviour of the bosonic superfluid may be overshadowed by the fermionic effects of localization and interaction. The superconducting energy gap in the wire may be suppressed and quasiparticles may be generated. However, we are not aware of any reason that would forbid QPS to be a physical reality. We thus assume that coherent QPS may take place, that it is characterized by a transition amplitude $E_{\mathrm{S}} / 2$ and that no quasiparticles are present ( $E_{\mathrm{S}}<\Delta, \Delta$ being the superconducting energy gap). On the

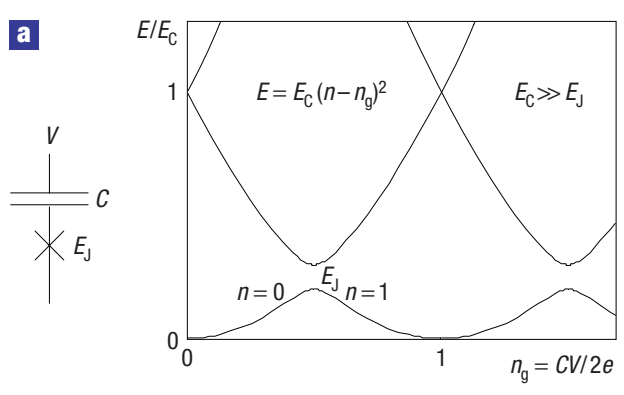

b

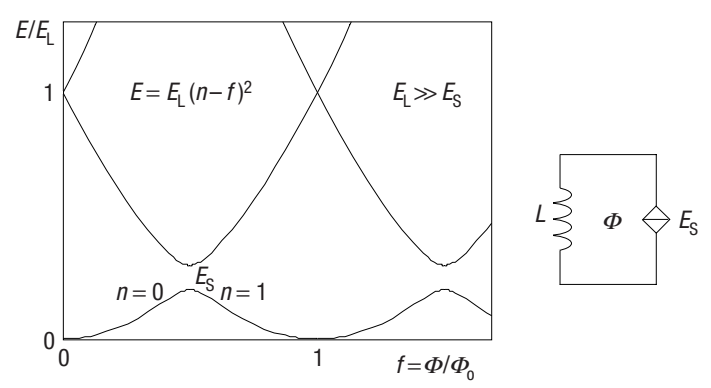

Figure 1 Circuit and energy dependence. a, A Cooper-pair box and b, a QPS qubit. The diamond-shaped symbol in the QPS qubit circuit represents the quantum phase-slip process. The capacitive energy is $E=E_{c}\left(n-n_{\mathrm{g}}\right)^{2}$ and the inductive energy is $E=E_{L}(f-n)^{2}$.

basis of Wentzel-Kramers-Brillouin (WKB)-type estimates ${ }^{3}$ and microscopic calculations ${ }^{7}$, we assume that the transition amplitude for QPS in practical superconducting nanowires of $1 \mu \mathrm{m}$ length can be as high as $E_{\mathrm{S}} / h=100 \mathrm{GHz}$.

Wires in which significant QPS occurs have large kinetic inductance $L^{\prime}$ and small capacitance $C^{\prime}$ per unit length. The plasmon phase velocity $c_{\mathrm{p}}=\left(L^{\prime} C^{\prime}\right)^{1 / 2}$ is of order $10^{5} \mathrm{~m} \mathrm{~s}^{-1}$ (refs 9 , 10) and the characteristic impedance $Z_{\mathrm{c}}=\left(L^{\prime} / C^{\prime}\right)^{1 / 2}$ is of order $100 \mathrm{k} \Omega$. Frequent QPSs are expected ${ }^{7}$ when $Z_{\mathrm{c}}>R_{\mathrm{q}}$, with $R_{\mathrm{q}} \equiv$ $h / 4 e^{2}=6.45 \mathrm{k} \Omega$. A next step in the understanding of QPS was made $e^{6}$ by considering a wire of finite length as a circuit element. 
If the rest of the circuit is modelled by a parallel resistance $R_{\mathrm{p}}$, it was predicted that the wire will show a quantum phase transition at $R_{\mathrm{p}}=R_{\mathrm{q}}$, being superconducting at $R_{\mathrm{p}}<R_{\mathrm{q}}$ and insulating otherwise. As predicted ${ }^{11}$, a Josephson junction shows qualitatively the same behaviour. The conclusion was then drawn ${ }^{6}$ that the lowenergy physics of QPSs reduces to that of a Josephson junction in the same circuit.

We explicitly demonstrate in this paper that the relation between Josephson tunnelling and QPS in circuits is more intriguing. They are dual to each other with respect to the exchange of the canonically conjugated quantum variables, phase and charge. This duality is exact, in contrast to the widely known ${ }^{12}$ approximate self-duality of Josephson junction circuits. Results of ref. 6 are reproduced by using exact duality and approximate self-duality. We use exact duality to describe the dynamical response of QPS in the limit of strong phase slip, where we reveal very favourable conditions for the observation of Bloch-type oscillations and of a resonance at the 'plasma' frequency.

The duality is developed in two steps. We first compare two simple circuits (Fig. 1). One is the Cooper-pair box ${ }^{13}$ with a Josephson junction that is voltage-biased with voltage $V$ through a capacitor $C$. In the other we introduce the QPS junction, an element that represents the phase-slip process with strength $E_{\mathrm{S}}$, in a closed loop with an inductor. This is the QPS flux qubit proposed in ref. 14, but not yet realized in practice. In the Cooper-pair box the charging energy depends parabolically on the induced charge $n_{\mathrm{g}}=C V / 2 e$, for each integer value of the Cooper-pair number $n$. The charging energy scale is given by $E_{\mathrm{C}}=(2 e)^{2} / 2 C$. Josephson coupling $E_{\mathrm{J}}$ mixes states with $n$ and $n+1$, lifting degeneracy at half-integer values of $n_{\mathrm{g}}$. The level splitting at this point equals $E_{\mathrm{J}}$ provided $E_{\mathrm{J}} \ll E_{\mathrm{C}}$. This is described by the following hamiltonian:

$$
H_{\mathrm{JJ}}=E_{\mathrm{C}}\left(n-n_{\mathrm{g}}\right)^{2}-\left(\frac{E_{\mathrm{J}}}{2} \sum_{n}|n+1\rangle\langle n|+\text { h.c. }\right),
$$

where h.c. represents the hermitian conjugate. In the QPS qubit on the right of Fig. 1 the inductive energy depends parabolically on the applied flux $\Phi$ with $f=\Phi / \Phi_{0}$ at each integer $n$ that now represents the fluxoid number in the loop. The scale for the inductive energy is $E_{L}=\Phi_{0}^{2} / 2 L$, where $L$ is the inductance of the loop. QPS coupling mixes states with adjacent fluxoid numbers and lifts degeneracy at half-integer values of $f$. The level splitting at this point equals $E_{S}$, provided $E_{\mathrm{S}} \ll E_{L}$. This implies the following hamiltonian:

$$
H_{\mathrm{QPS}}=E_{L}(n-f)^{2}-\left(\frac{E_{\mathrm{S}}}{2} \sum_{n}|n+1\rangle\langle n|+\text { h.c. }\right) \text {. }
$$

The diagram as drawn for the Cooper-pair box is only valid when $E_{\mathrm{C}} \gg E_{\mathrm{J}}$, where charge is the relevant quantum number. In analogy, the diagram for the QPS qubit is only valid when $E_{L} \gg E_{S}$, the limit of weak phase slip. Here, phase is the relevant quantum number. The above hamiltonians are obviously equivalent with respect to the exchange

$$
E_{L} \Leftrightarrow E_{\mathrm{C}} ; E_{\mathrm{J}} \Leftrightarrow E_{\mathrm{S}} ; n_{\mathrm{g}} \Leftrightarrow f .
$$

At this stage we have not yet made use of the fact that charge and phase are canonically conjugated quantum variables.

We will now derive the exact duality from this fact. We start by considering a Josephson junction in a linear circuit with either a voltage or a current source (Fig. 2). Any linear circuit can be presented by an equivalent (frequency-dependent) resistor in series with (for voltage bias) or parallel to (for current bias) the junction. A quantum variable describing the circuit is either the phase across the junction or the continuous number of Cooper

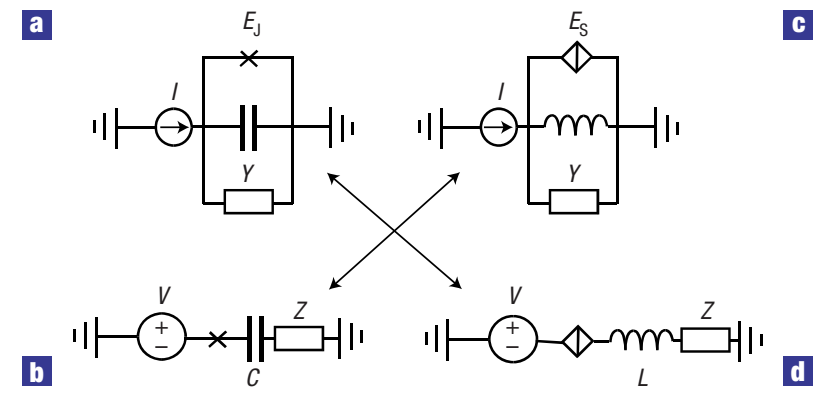

Figure 2 Dual equivalence of Josephson and QPS junctions in circuits.

a, Current-biased Josephson junction; b, voltage-biased Josephson junction; c, current-biased QPS junction; d, voltage-biased QPS junction. Circuit a is the exact dual of circuit $\mathbf{d}$; circuit $\mathbf{b}$ is the exact dual of circuit $\mathbf{c}$.

pairs transferred. Those are canonically conjugated variables so that the corresponding operators of charge $(\hat{q})$ and phase $(\hat{\phi})$ satisfy $[\hat{q}, \hat{\phi}]=-i$. In the spirit of the Caldeira-Legett approach ${ }^{15}$, the circuit can be described with the following hamiltonian:

$$
\hat{H}=E_{\mathrm{C}} \hat{q}^{2}-E_{\mathrm{J}} \cos \hat{\phi}+\hat{H}_{\text {env }}+\hat{H}_{\text {coupling }} .
$$

Here, $\hat{H}_{\text {env }}$ represents boson-like environment modes, and the coupling term $\hat{H}_{\text {coupling }}$ is different for voltage or current bias,

$$
\hat{H}_{\text {coupling }}=\left\{\begin{array}{ll}
\frac{\Phi_{0}}{2 \pi}\left(I-\hat{I}_{\mathrm{r}}\right) \hat{\phi} & \text { for current bias } \\
-2 e\left(V-\hat{V}_{\mathrm{r}}\right) \hat{q} & \text { for voltage bias }
\end{array} .\right.
$$

The operators $\hat{I}_{\mathrm{r}}$ and $\hat{V}_{\mathrm{r}}$ present current and voltage fluctuations, respectively, in the effective resistor and are linear combinations of the environment bosons. The coefficients in these linear combinations are fixed to reproduce the response function of the environment:

$$
\begin{aligned}
\hat{I}_{\mathrm{r}}(\omega) & =\frac{\hbar}{2 e}(-i \omega) Y(\omega) \hat{\phi}(\omega) \\
\hat{V}_{\mathrm{r}}(\omega) & =2 e(-i \omega) Z(\omega) \hat{q}(\omega) .
\end{aligned}
$$

Here $Z(\omega)$ is the frequency-dependent impedance of a serial resistor, $Y(\omega)$ the admittance of a parallel resistor and $\hbar$ is the reduced Planck constant.

For the hamiltonian of a QPS junction in a circuit, we use the inductive and QPS energies from (1) to obtain

$$
\hat{H}=\frac{E_{L}}{(2 \pi)^{2}} \hat{\phi}^{2}-E_{\mathrm{S}} \cos (2 \pi \hat{q})+\hat{H}_{\text {env }}+\hat{H}_{\text {coupling }} .
$$

Let us now consider the effect of the canonical transformation $(\hat{q}, \hat{\phi}) \rightarrow(-\hat{\phi} / 2 \pi, 2 \pi \hat{q})$ on the hamiltonian (2). Obviously, that transformation does not change the commutation relations. We see that it transforms the QPS hamiltonian into the Josephson hamiltonian with the following parameters:

$$
E_{\mathrm{S}} \rightarrow E_{\mathrm{J}} ; E_{L} \rightarrow E_{\mathrm{C}} ; I \leftrightarrow R_{\mathrm{q}}^{-1} V ; Y(\omega) \leftrightarrow R_{\mathrm{q}}^{-2} Z(\omega) .
$$

Double-sided arrows mean that the transformation exchanges current and voltage bias and series and parallel resistors (Fig. 2). This is the main exact result of our work. These duality relations allow us to exactly map any known result concerning transport characteristics of Josephson junctions in a circuit to dual transport characteristics of QPS junctions in the dual circuit. 
The circuits of Fig. 1 were in the regimes $E_{\mathrm{C}} \gg E_{\mathrm{J}}$ and $E_{L} \gg E_{\mathrm{S}}$. Now let us turn to the opposite regimes for the above hamiltonians. For a Josephson junction, this limit is achieved at $E_{\mathrm{J}} \gg E_{\mathrm{C}}$, where the phase $\phi$ is a well-defined variable. Of particular interest is the dynamics of the classical Josephson junction as described by the resistively shunted junction model

$$
I(t)=I_{\mathrm{c}} \sin \phi+\frac{\Phi_{0}}{2 \pi}\left(C \frac{\mathrm{d}^{2} \phi}{\mathrm{d} t^{2}}+\frac{1}{R} \frac{\mathrm{d} \phi}{\mathrm{d} t}\right)
$$

that describes a motion in a slanted sinusoidal ('washboard') potential at time $t$ (ref. 1). Time-independent solutions correspond to the zero-voltage state of the junction where the phase is trapped in one of the potential minima. These minima exist provided the drive current $I$ does not exceed the critical current of the junction $I_{\mathrm{c}}=2 \pi E_{\mathrm{J}} / \Phi_{0}$. To obtain the dual counterpart of this equation for the voltage-biased QPS junction we make use of the transformation (3) that relates the circuits (a) and (d) from Fig. 2. The conductor that shunts the Josephson junction is transformed into an external resistor $R$ in series with the QPS junction. Equation (4) becomes an equation for the charge variable $q$,

$$
V(t)=V_{\mathrm{c}} \sin (2 \pi q)+2 e\left(L \frac{\mathrm{d}^{2} q}{\mathrm{~d} t^{2}}+R \frac{\mathrm{d} q}{\mathrm{~d} t}\right),
$$

where the terms on the right give the voltage drops over QPS junction, inductance and resistor, respectively. This expression is valid in the limit of large phase-slip amplitudes $E_{S} \gg E_{L}$ where the charge is a well-defined quantum variable. Here we have a washboard potential in the charge variable. The charge may be trapped in potential minima resulting in an insulating zero-current state provided the bias voltage $V$ does not exceed the critical voltage

$$
V_{\mathrm{c}}=\frac{2 \pi E_{\mathrm{s}}}{2 e} .
$$

For the Josephson junction in the classical regime, the response to small signals follows the kinetic inductance $L_{\text {kin }}=\Phi_{0} /\left(2 \pi I_{\mathrm{c}} \cos \phi\right)$. Similarly for QPS junctions the response is according to a kinetic capacitance

$$
C_{\mathrm{kin}}=\frac{2 e}{2 \pi V_{\mathrm{c}} \cos 2 \pi q} .
$$

The geometry of Josephson tunnel junctions implies a shunt capacitance. The combination of kinetic inductance and shunt capacitance leads to a resonance at the so-called plasma frequency $\omega_{\mathrm{p}}=\left(2 E_{\mathrm{J}} E_{\mathrm{C}}\right)^{1 / 2}$. Because $E_{\mathrm{J}}$ scales with the area and $E_{\mathrm{C}}$ scales inversely with it, $\omega_{\mathrm{p}}$ is independent of area for a homogeneous barrier. For a QPS junction, the natural geometry is a wire with considerable kinetic inductance. Now, the resonance provided by kinetic capacitance (6) and inductance has a frequency

$$
\omega_{\mathrm{p}}=\sqrt{2 E_{\mathrm{S}} E_{L}} .
$$

As $E_{S}$ is proportional and $E_{L}$ is inversely proportional to the wire length, the plasma frequency is constant when the length is changed. However, in a lithographically fabricated nanowire it will be easy to lower $\omega_{\mathrm{p}}$ by adding a wider section of wire that lowers $E_{L}$ without increasing $E_{S}$ (Fig. 3). It is interesting to note that moving from long wires to short wires, the ratio $E_{S} / E_{L}$ decreases in the same way as the decrease of $E_{\mathrm{J}} / E_{\mathrm{C}}$ when going from large-area to smallarea Josephson junctions. Whereas in the latter case a transition is made from superconducting to insulating response, the wires may be insulating for long lengths and recover superconductivity at shorter lengths.
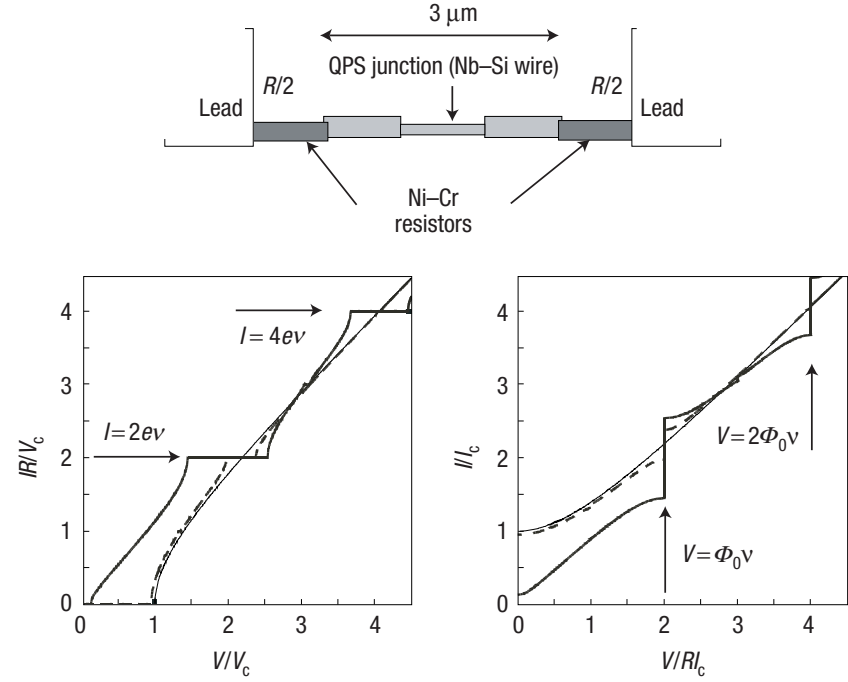

Figure 3 Shapiro steps at constant current in a QPS junction. Top: The junction must be embedded in a highly resistive environment. Parasitic capacitances are sufficiently small for the design given. Left: QPS junction. The parameters correspond to $Q=0.5,2 \pi v=\omega_{p}, V_{\text {a.c. }} / V_{\mathrm{c}}=0,1,5$ for the thick solid, dashed and thin solid curves respectively. With $E_{\mathrm{S}} / h=120 \mathrm{GHz}$ and $E_{L} / h=30 \mathrm{GHz}$ the plasma frequency is $\omega_{\mathrm{p}} / 2 \pi=85 \mathrm{GHz}$ and the critical voltage is $V_{\mathrm{c}}=1.56 \mathrm{mV}$; $R=115 \mathrm{k} \Omega$. Right: Voltage Shapiro steps in the dual Josephson circuit with equivalent parameters.

The high-frequency environmental impedance of a submicrometre junction is usually determined by the geometric capacitance and inductance of nearby wiring and, without special precautions, has a value around $300 \Omega \ll R_{\mathrm{q}}$. For a QPS junction this natural impedance leads to a high quality factor of the plasma oscillations given by

$$
Q_{\mathrm{QPS}}^{2}=\beta_{L}=2 \pi \frac{V_{\mathrm{c}}}{2 e} \frac{L}{R^{2}}=2 \pi^{2} \frac{E_{\mathrm{S}}}{E_{L}}\left(\frac{R_{\mathrm{q}}}{R}\right)^{2} .
$$

Here $\beta_{L}$ is the equivalent of the well-known McCumber parameter $\beta_{\mathrm{C}}$ for Josephson junctions. Using duality, we predict a strongly nonlinear hysteretic response to relatively small resonant signals $V_{\text {a.c. }} / V_{\mathrm{c}} \sim 1 / \mathrm{Q}_{\mathrm{QPS}}, V_{\text {a.c. }}$ being the amplitude of the applied radiofrequency (RF) signal. The dual effect was investigated for a precision quantum measurement with Josephson junctions ${ }^{16}$.

The most far-reaching application of QPS junctions might be in a fundamental standard of current, dual to the well-known Josephson voltage standard. Equation (5) can be used to calculate the transport in the series circuit of QPS junction, inductance and resistance. When an RF voltage is applied, the equivalent of Shapiro steps will occur in the form of plateaus at constant current levels

$$
I_{n}=n 2 e v,
$$

where $v$ is the RF frequency. For $n=1$ and $v=80 \mathrm{GHz}$, as used in Josephson voltage standards, the current level would be $26 \mathrm{nA}$. High $Q_{\mathrm{QPS}}$ is unfavourable for this application as it gives rise to a hysteretic current-voltage characteristic, unrealistically high frequencies $\sim Q_{\mathrm{QPS}}$ and modulation amplitudes $\sim Q_{\mathrm{QPS}}^{2}$. To achieve a practical device, the circuit should be made non-hysteretic by the use of a sufficiently high series resistance. As $E_{S} / E_{L}$ cannot be smaller than about 4 to guarantee that charge, as the relevant 
quantum variable, is well defined, the series resistance has to be at least $60 \mathrm{k} \Omega$. In Fig. 3 we give current-voltage characteristics for a circuit with quality factor $Q=0.5$, driven at the plasma frequency. Realistic parameters are given in the figure. To fabricate the QPS wire as well as two series resistors of $60 \mathrm{k} \Omega$ each, without shunting by parasitic capacitances, is challenging but seems possible with state-of-the-art electron-beam lithography. Many devices could be put in parallel on a chip. The current standard would have to be operated at low temperatures as the presence of quasiparticles leads to a conduction channel parallel to the QPS junction, which would reduce the precision.

The QPS junction at $E_{\mathrm{S}} \gg E_{L}$ and the Josephson junction at $E_{\mathrm{C}} \gg E_{\mathrm{J}}$ have in common that the charge is the relevant quantum variable. For Josephson junctions in the charging regime, Bloch oscillations were predicted ${ }^{17,18}$ and observed ${ }^{19}$. This system is often considered as dual to the classical Josephson junction, because of the crossover from phase to charge as the quantum variable. However, there is no strict duality as shown for Josephson and QPS. Interestingly, the weak QPS regime with $E_{L} \gg E_{S}$ and the classical Josephson junction share the phase as the quantum variable.

Received 22 November 2005; accepted 18 January 2006; published 12 February 2006.

\section{References}

1. Tinkham, M. Introduction to Superconductivity 2nd edn 288 (McGraw-Hill, New York, 1996).

2. Giordano, N. Evidence for macroscopic quantum tunneling in one-dimensional superconductors. Phys. Rev. Lett. 61, 2137-2140 (1988).

3. Lau, C. N., Markovic, N., Bockrath, M., Bezryadin, A. \& Tinkham, M. Quantum phase slips in superconducting nanowires. Phys. Rev. Lett. 87, 217003 (2001).

4. Bezryadin, A., Lau, C. N. \& Tinkham, M. Quantum suppression of superconductivity in ultrathin nanowires. Nature 404, 971-974 (1999).
5. Bollinger, A. T., Rogachev, A. \& Bezryadin, A. Coulomb blockade in the insulating regime of short superconducting nanowires. Preprint at $<$ http://arxiv.org/abs/cond- mat/0508300 $>$ (2005).

6. Büchler, H. P., Geshkenbein, V. B. \& Blatter, G. Quantum fluctuations in thin superconducting wires of finite length. Phys. Rev. Lett. 92, 067007 (2004).

7. Zaikin, A. D., Golubev, D. S., van Otterlo, A. \& Zimanyi, G. T. Quantum phase slips and transport in ultrathin superconducting wires. Phys. Rev. Lett. 78, 1552-1555 (1997).

8. Golubev, D. S. \& Zaikin, A. D. Quantum tunneling of the order parameter in superconducting nanowires. Phys. Rev. B 64, 014504 (2001).

9. Kulik, I. O. Frequency dependence of the penetration depth of an electric field in a superconductor Fiz. Nizk. Temp. 5, 1391 (1979); English translation: Sov. J. Low Temp. Phys. 5, 656-662 (1979).

10. Mooij, J. E. \& Schön, G. Propagating plasma mode in thin superconducting filaments. Phys. Rev, Lett. 55, 114-117 (1985).

11. Schmid, A. Diffusion and localization in a dissipative quantum system. Phys. Rev. Lett. 51, 1506-1509 (1983).

12. Schön, G. \& Zaikin, A. D. Quantum coherent effects, phase transitions, and the dissipative dynamics of ultra small tunnel junctions. Phys. Rep. 198, 237-412 (1990).

13. Bouchiat, V., Vion, D., Joyez, P., Esteve, D. \& Devoret, M. H. Quantum coherence with a single Cooper pair. Phys. Scr. T 76, 165-170 (1998).

14. Mooij, J. E. \& Harmans, C. J. P. M. Phase-slip flux qubits. New J. Phys. 7, 219 (2005)

15. Caldeira, A. O. \& Leggett, A. J. Quantum tunnelling in a dissipative system. Ann. Phys. 149, 374-456 (1983).

16. Siddiqi, I. et al. Direct observation of dynamical bifurcation between two driven oscillation states of a Josephson junction. Phys. Rev. Lett. 94, 027005 (2005).

17. Averin, D. V., Zorin, A. B. \& Likharev, K. K. Bloch oscillations in small Josephson junctions. Sov. Phys. JETP 61, 407-413 (1985).

18. Likharev, K. K. \& Zorin, A. B. Theory of the Bloch-wave oscillations in small Josephson junctions. J. Low Temp. Phys. 59, 347-382 (1985).

19. Kuzmin, L. \& Haviland, D. B. Observation of the Bloch oscillations in an ultrasmall Josephson junction. Phys. Rev. Lett. 67, 2890-2893 (1991).

\section{Acknowledgements}

We thank L. Levitov, L. Glazman, T. P. Orlando, C. J. P. M. Harmans, A. D. Zaikin and A. Bezryadin for discussions. Our research is supported by FOM, NanoNed and EuroSQIP.

Correspondence and requests for materials should be addressed to J.E.M.

Competing financial interests

The authors declare that they have no competing financial interests.

Reprints and permission information is available online at http://npg.nature.com/reprintsandpermissions/ 